\title{
Statistics for Data Analysis Using Microsoft Excel
}

\author{
Chief Assist. Prof. Dr. Svetlana Todorova \\ University of Economics - Varna, Varna, Bulgaria \\ svetlana.todorova@ue-varna.bg
}

\begin{abstract}
Today business research is based on the manipulation of large amounts of data from the Internet, company sources, public sources, and others. Business analytics must extract useful information from a big data set and to make a decision. Whether you are analyzing a client's data or company's data to make decisions, your tools have to be able to handle the tasks you perform with that information. The goal of this paper is to illustrate the use of Excel's Analysis ToolPak for developing complex statistical analysis. MS Excel is widespread, user-friendly, easy to learn, and powerful. Therefore, the purpose of this paper is to show how free Analysis ToolPak add-in works and what it can do. In one hand Analysis ToolPak is powerful, but in the other hand it has some weaknesses that we should be aware of.
\end{abstract}

Keywords: Statistics, Data Analysis, Excel, Analysis ToolPak

JEL Code:C880; doi:10.36997/IJUSV-ESS/2019.8.2.68

\section{Въведение}

Съвременните бизнес изследвания се основават на извличане на големи масиви от данни от Интернет, фирмени източници, публични източници, статистически справочници и др. Статистическият анализ на данни, които включват много на брой променливи и наблюдения, изисква от изследователя задълбочени знания за статистическите методи. В повечето случаи обработката на такива данни се реализира с помощта на специализиран софтуерен продукт. В широк смисъл статистически е всеки софтуер, който е в състояние да обработва съвкупностна (статистическа) информация и притежава възможности за изчисляване на обобщаващи характеристики. В тесен смисъл статистически е всеки софтуер, който притежава възможности за изчисляване на обобщаващи характеристики от основните дялове на теоретичната статистика - анализ на емпирични разпределения, статистически заключения, анализ на корелационни зависимости и др. (Хаджиев, 2009, p. 9-10).

MS Excel е широко използван софтуер за статистически анализ на данни, представени във вид на електронни таблици. Намира приложение и при обработка на големи масиви от данни. Той предоставя възможности за графично изобразяване на данните и дава възможност за реализиране както на основни статистически функции, така и на комплексен статистически анализ с модула Data Analysis. MS Excel e добър избор, защото той е леснодостьпен, спестява време, осигурява лесен обмен на данни с други приложения, визуализира много добре графично и таблично крайните резултати и др.

Всяка следваща версия на MS Excel добавя нови възможности за графично представяне и интерпретация на данни: Box \& Whisker, Histogram, Scatter (X, Y), Trendline и др. В MS Excel съществуват две възможности за приложение на статистически методи: основни статистически функции и модула Data Analysis. Според предназначението си функциите са класифицирани в 13 категории. Всяка функция е достьпна от раздел Formulas, група Statistical Function (Сълов и др., 2017 р. 218-222). Обработката на данните по този начин се извършва чрез вградени функции, които участват във формули за определяне на съдържанието на дадена клетка. Модулът Data Analysis съдържа 19 инструмента за различни статистически анализи и тестове, които позволяват извършването на един по-комплексен статистически анализ.

Наред с новите диаграми, Microsoft постоянно обновява и статистическите функции на MS Excel. Те стават все по-точни и все повече задоволяват нарастващото потребителско търсене. От друга страна функционалността и потребителският интерфейс на Analysis 
ToolPak са същите от десетилетия. Любопитно е, че предвид все по-голямото внимание на Microsoft към анализа на данни и съсредоточаването му върху подобряване на функциите и добавянето на нови диаграми за анализ на данни, защо запазва Analysis ToolPak такъв, какъвто е.

Целта на този доклад е, въпреки някои от слабите стрни на Analysis ToolPak, да ce илюстрира лекотата и достьпността на Analysis ToolPak за разработване на комплексен статистически анализ.

\section{1. Инсталиране на модула Data Analysis ToolPak на MS Excel}

В настоящата статия се ограничаваме до описание на възможностите на специализирания модул Data Analysis в MS Excel. Той допълва наличната функционалност на електронната таблица, като добавя към възможностите й да представя и трансформира динамични редове и мощни възможности за извършване на статистически анализ (Славева и др., 2016, p. 522-523). Основни статистически анализи и тестове в Data Analysis ca: описателна статистика, дисперсионен анализ, регресионен анализ, корелационен анализ, метод на плъзгащите се средни, проверка на статистически хипотези и други.

Преди да се започне с използването на Analysis ToolPak, той трябва да бъде зареден, чрез add-ins: За целта се избира от „Опциите” на Excel (в Excel 2010 или по-нови версии):

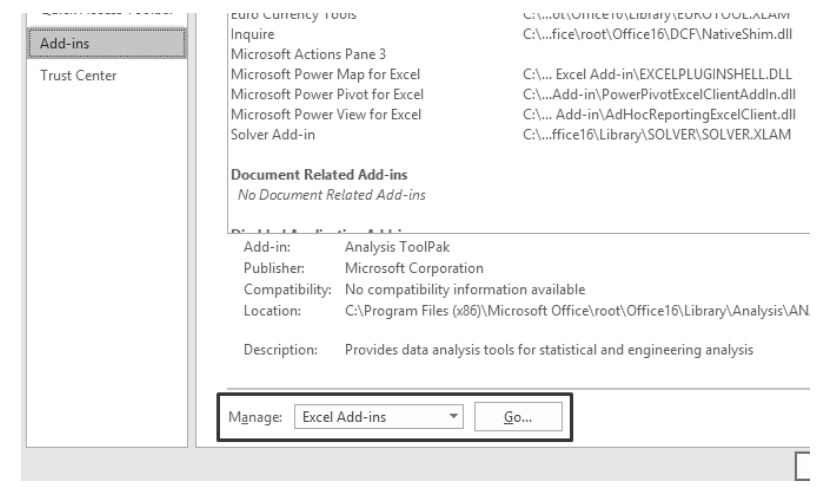

Add-ins, Gо и след това Add-ins ToolPak:

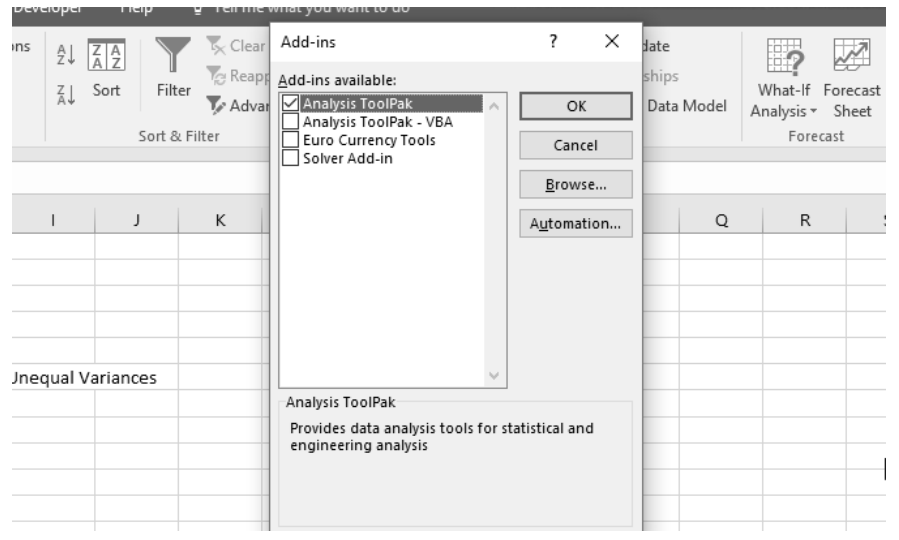

Трябва да се обърне внимание, че има и Analysis ToolPak - VBA. Версията VBA включва Visual Basic за разработване на приложения на основата на създаване на макроси. Ако не планирате да автоматизирате Analysis ToolPak c VBA, той няма нужда да се инсталира.

При правилно добавяне на Analysis ToolPak в Excel, на лентата с команди в раздела Data се появява бутон Data Analysis: 

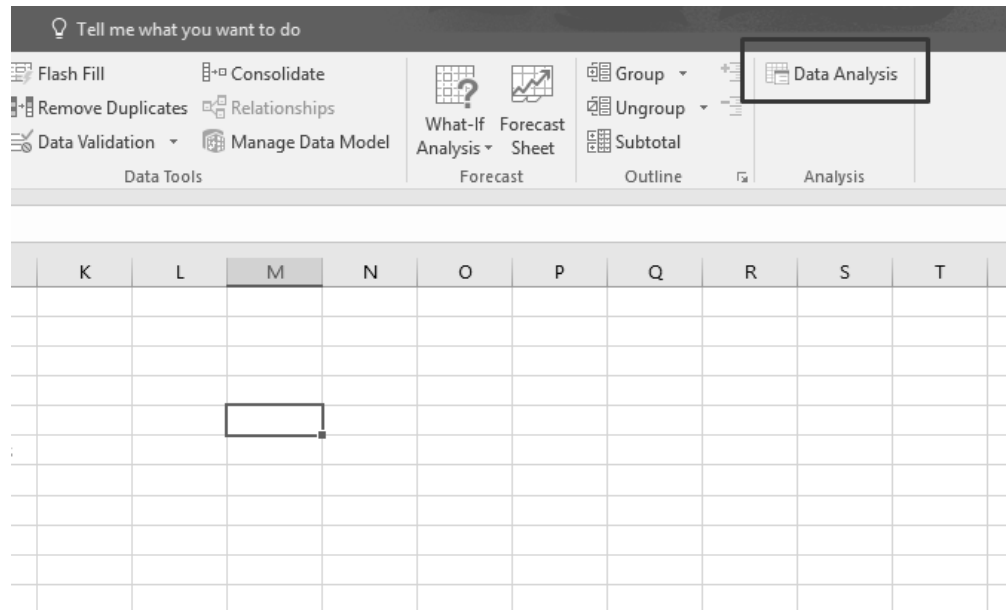

След като модулът за анализ на данни е добавен се появява Data Analysis в крайната дясна част на лентата. При избор на Data Analysis ще се отвори диалоговия прозорец, който предлага достьп до различни статистически анализи и тестове:

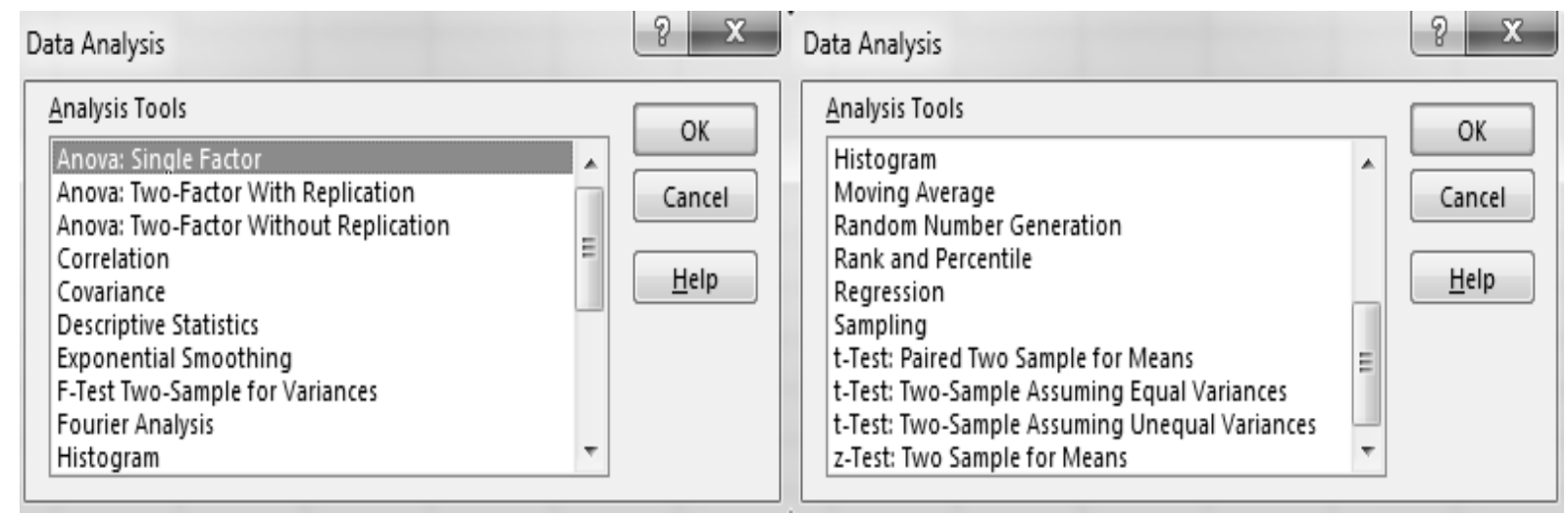

\section{2. Описателна статистика в MS Excel}

Независимо какъв статистически анализ или тест ще се провежда, почти винаги първо е необходимо да се получат обобщаващи числови характеристики, графики и таблици (описателната статистика). Това дава информация за средната, медианата, модата, стандартното отклонение, дисперсията, асиметрията и ексцеца на емпиричното разпределение на данните, с които се разполага. Изпълнението на описателната статистика в MS Excel е лесно и бързо. Избира се Data Analysis в раздела Data и след това Descriptive Statistics като данните се въвеждат чрез маркиране. Може да се окаже на Excel дали данни имат етикети, дали резултатите от анализа да са на нов лист или на същия и други опции:

Data Analysis
\begin{tabular}{|l|l|l|}
\hline Analysis Tools & OK \\
\hline $\begin{array}{l}\text { Anova: Single Factor } \\
\text { Anova: Two-Factor With Replication } \\
\text { Anova: Two-Factor Without Replication } \\
\text { Correlation } \\
\text { Covariance }\end{array}$ \\
\hline $\begin{array}{l}\text { Descriptive Statistics } \\
\text { Exponential Smoothing } \\
\text { F-Test Two-Sample for Variances } \\
\text { Fourier Analysis } \\
\text { Histogram }\end{array}$ \\
\hline
\end{tabular}

\begin{tabular}{|c|c|c|c|}
\hline \multicolumn{3}{|l|}{ Descriptive Statistics } & $8 B$ \\
\hline \multicolumn{4}{|l|}{ Input } \\
\hline Input Range: & \multicolumn{2}{|c|}{ SDS2:SDS30 睓国 } & \\
\hline Grouped By: & \multirow{2}{*}{\multicolumn{2}{|c|}{$\begin{array}{l}\text { (1) Columns } \\
\text { Rows }\end{array}$}} & Cancel \\
\hline \multicolumn{2}{|l|}{ Labels in first row } & & \\
\hline \multicolumn{4}{|l|}{ Output options } \\
\hline O utput Range: & & 国 & \\
\hline \multicolumn{4}{|l|}{ (- New Worksheet Ply: } \\
\hline \multicolumn{4}{|l|}{ New $\underline{\text { workbook }}$} \\
\hline \multicolumn{4}{|l|}{ D Summary statistics } \\
\hline \multicolumn{4}{|l|}{$\nabla$ Confidence Level for Mean: } \\
\hline$\square$ Kth Largest: & 1 & & \\
\hline$\square$ Kth Smallest: & 1 & & \\
\hline
\end{tabular}


Струва си да се спомене, че ако се избере Confidence Level (равнище на значимост), при проследяване на резултатите от анализа на последния ред се появява число, чиято стойност трябва да се извади и прибави към средната, за да се получи $95 \%$ доверителен интервал (по подразбиране е заложено 95\% равнище на значимост). Следователно, за да се получи доверителен интервал, се нужни допълнителни изчисления.

Опцията Histogram в Analysis ToolPak позволява да се създаде честотна таблица и съпьтстваща диаграма - хистограма. В диалоговия прозорец на Histogram се изисква не само диапазона на данни, но също така и диапазона Bins, но за него няма стойности по подразбиране. Потребителят избира Bins като го поставя някъде в работния лист. Това всъщност е горната граница на интервалите при групирани данни. По подразбиране резултатите се появяват на нов работен лист и включват таблицата с честотните разпределения и съответната диаграма. За да се направи така, че стълбчетата на хистограмата да са точно долепени едно до друго, се избира форматиране на диаграмата и Gap Width $=0$. Трябва да се обърне внимание, че слабостите на хистограмата от Analysis ToolPak могат да се преудолеят ако се използва новата вградена диаграма, която за първи пьт се въвежда в Excel 2016. За да създадете хистограма в Excel 2016, трябва само да се маркират данни, да се избере от раздела Вмъкване на групата Графики и да се намери Хистограма. Получава се хистограмата без да е необходимо дефиниране на долна или горна граница на интервалите и не се налага редактирането и. Добрата новина e, че Box \& Whisker plot е нов вграден тип диаграма, представена също в Excel 2016. За да създадете Box \& Whisker plot, се следват същите стьпки като се избира Box \& Whisker. Освен това всяка диаграма в MS Excel може да се персонализира чрез Дизайн / Формат. Статистическите диаграмите позволяват по-добро възприемане на зависимостите, емпиричното разпределение или тенденциите в развитието.

За представяне на описателната статистика използваме реални данни за средната месечна брутна работна заплата по области за 2017 г. Резултататите от анализа могат да са на нов работен лист (по подразбиране) или на същия работен лист ако се зададе поле, в което да се поместят. Средната месечна работна заплата според средната аритметична е 840 лв., като най-ниска е в област Видин - 686 лв. и най-висока в София - град - 1433 лв. Епиричното разпределение е несиметрично, с ясна изразена положителна асиметрия (Histogram \& Boxplot). Това показва, че за определяне центръра на разпределение е поподходящо да се използва медианата, която е 795 лв. Това означава, че 50\% от населението е с месена заплата по-малка от 795 лв. и 50\% от населението е със заплата по-висока от 795 лв. Средната месечна работна заплата в София-град е екстремална стойност (Boxplot) по отношение на средните работни заплати в другите области и не показва общото и типичното за съвкупността. Това е и причината, изчислената средна работна заплата, чрез средната аритметична да е значително по-висока по стойност в сравнение с медианата:

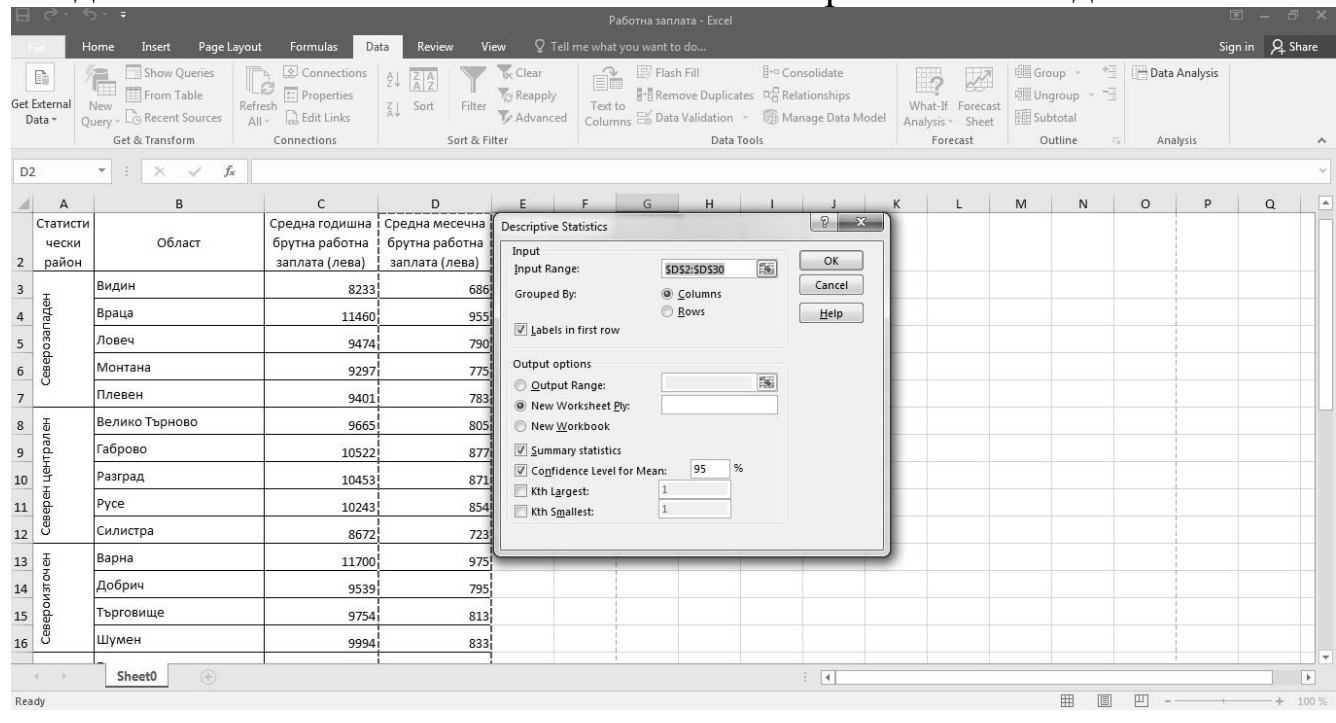




\section{IZVESTIA JOURNAL OF THE UNION OF SCIENTISTS - VARNA}

\begin{tabular}{|l|r|}
\hline \multicolumn{2}{|l|}{ Средна месечна брутна работна заплата (лева) } \\
\hline & \\
\hline Mean & 840.4047619 \\
\hline Standard Error & 27.74880954 \\
\hline Median & 794.5416667 \\
\hline Mode & \#N/A \\
\hline Standard Deviation & 146.8328984 \\
\hline Sample Variance & 21559.90006 \\
\hline Kurtosis & 9.364579 \\
\hline Skewness & 2.626338851 \\
\hline Range & 747.1666667 \\
\hline Minimum & 686.0833333 \\
\hline Maximum & 1433.25 \\
\hline Sum & 23531.33333 \\
\hline Count & 28 \\
\hline Confidence Level(95.0\%) & 56.93585355 \\
\hline
\end{tabular}

\section{3. Корелационен и регресионен анализ в MS Excel}

Изчисляването на корелационни коефициенти в Excel е бързо и удобно. Трябва само да изберете Correlation и да маркирате променливите, за които ще се изчислят корелационните коефициенти. Предимство на инструмента за корелационен анализ в Analysis ToolPak e, че вместо функцията, която се използва само за две променливи, тук може да маркарират повече променливи и като резултат се получава корелационна матрица с изчислените коефициенти за всички двойки променливи. Това дава обща картина между кои променливи съществува положителна и между кои отрицателна зависимост. Трябва да се отбележи, обаче, че Excel изчислява само корелационния коефициент на Браве-Пирсън, който се прилага при линейни зависимости. Този коефициент на корелация се променя в границите -1 и 1. Ако абсолютната му стойност е близка до 1, това означава, че между променливите съществува силна връзка, а ако е близък до 0, то връзката е слаба.

Описаният корелационен анализ се реализира чрез пример за паричния доход на лице от домакинство и потреблението на основни хранителни продукти на лице от домакинство в натурални измерители по децилни групи за 2018 г. Изчисления корелационен коефициент между паричния доход и хляб и тестени изделия е -0,88. Това показва силна и обратна зависимост между променливите. При увеличаване на дохода, потреблението на хляб и тестени изделия като малоценна стока намалява, защото хората започват да купуват поскъпи и по-полезни хранителни продукти като риба, пресни плодове, зеленчуци и месо. Коефициентите на корелация между рибата, пресните плодове, зеленчуци и месото с паричния доход показват силна положителна зависимост, т.е. с увеличаване на дохода, тяхното потребление расте. За преориентацията в потреблението при увеличаване на дохода говорят и отрицателните корелационни коефициенти на хляба и тестените продукти с всички останали хранителни продукти. При намаляване на потреблението на хляб хората започват да потребяват повече от нормалните стоки:

\begin{tabular}{|c|c|c|c|c|c|c|}
\hline & $\begin{array}{c}\text { Паричен доход, } \\
\text { средно на лице - } \\
\text { левове }\end{array}$ & $\begin{array}{c}\text { Хляби и } \\
\text { тестени } \\
\text { изделия - } \\
\text { ке. }\end{array}$ & $\begin{array}{c}\text { Meco - } \\
\text { Ke }\end{array}$ & $\begin{array}{l}\text { Рибаи } \\
\text { рибни } \\
\text { продук } \\
\text { ти-ке } \\
\end{array}$ & $\begin{array}{c}\text { Плодове - } \\
\text { пресни и } \\
\text { замразени } \\
- \text {-ке } \\
\end{array}$ & $\begin{array}{c}\text { Зеленчуци - } \\
\text { пресни и } \\
\text { замразени - } \\
\text { ке } \\
\end{array}$ \\
\hline $\begin{array}{l}\text { Паричен доход, } \\
\text { средно на лице - } \\
\text { левове }\end{array}$ & 1 & & & & & \\
\hline $\begin{array}{l}\text { Хляб и тестени } \\
\text { изделия - кг. }\end{array}$ & -0.882373341 & 1 & & & & \\
\hline Мeco - kr & 0.672479743 & -0.61520942 & 1 & & & \\
\hline $\begin{array}{l}\text { Риба и рибни } \\
\text { продукти - кг }\end{array}$ & 0.959197318 & -0.83847332 & 0.80952 & 1 & & \\
\hline $\begin{array}{l}\text { Плодове - пресни и } \\
\text { замразени - кг }\end{array}$ & 0.876718988 & -0.71727976 & 0.904456 & 0.924294 & 1 & \\
\hline $\begin{array}{l}\text { Зеленчуци - пресни и } \\
\text { замразени - кг }\end{array}$ & 0.803511883 & -0.65707538 & 0.948255 & 0.886495 & 0.98537474 & 1 \\
\hline
\end{tabular}


Регресионният анализ е един от най-често използваните статистически анализи в бизнес изследванията, а изчисления при него са много трудоемки ако не се използва статистически софтуер. B MS Excel обаче, регресионният анализ се реализира бързо и лесно. Пьрво трябва да се избере Regression и след това внимателно да се попълнят полетата и да се изберат променливите. Необходимо е да се определи коя е зависимата и коя е независимата променлива или независимите променливи, а въвеждането им става чрез маркиране на данните. Резултатьт от приложения регресионен анализ по подразбиране е на нова страница и включва таблица с обобщаващи числови характеристики, ANOVA за проверка на адекватността на целия регресионен модел и трета таблица с информация за отделните коефициенти на регресия. Една любопитна характеристика е, че автоматично се получават два доверителни интервала за параметрите на регресионния модел, независимо дали сте поставили отметка в полето за равнище на значимост. Оказва се, че първият доверителен интервал винаги е с 95\% равнище на значимост, а вторият е с равнище на значимост по избор на потребителя, например 90\%, но само ако поставите отметка в полето за равнище на значиммост:

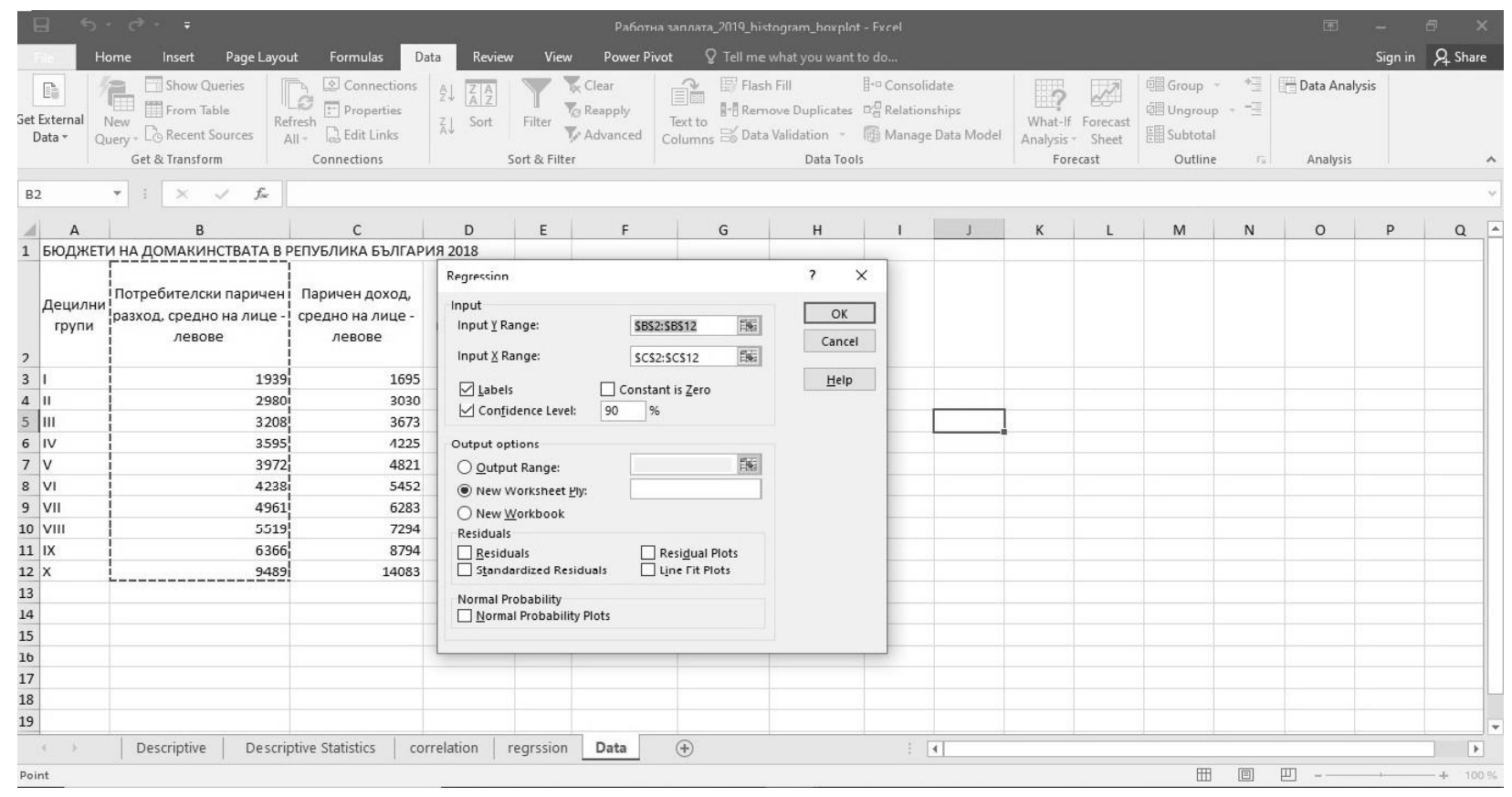

Регресионният анализ се реализира, чрез модел на потребителска функция на основата на данни за потребителския паричен разход и паричния доход средно на лице от домакинство за 2018 г. Зависимата променлива е потреблението, а независимата дохода. Оценките на параметрите на модела са поместени в третата таблица. Те са статистически значими и показват, че доходът е статически значим фактор при определяне на потреблението. В същата насока е и коефициента на детерминация (99,8\%), който показва, че $99,8 \%$ от вариацията в потреблението се определя от вариациата в дохода. Оценката на свободния член в регресионното уравнение $(1038,70)$ се нарича още автономно потребление и е онази част от потреблението, която не зависи от разполагаемния доход и показва размера на потреблението дори при нулев доход. Тази стойност може да е отправна точка при определяне на минималната и средната работни заплати от правителството. Оценката на параметъра пред независимита променлива $(0,60)$ е известна като пределната стойност на потребление и разкрива, че при всеки допълнителен лев доход за потребление се изразходват 60 ст.: 


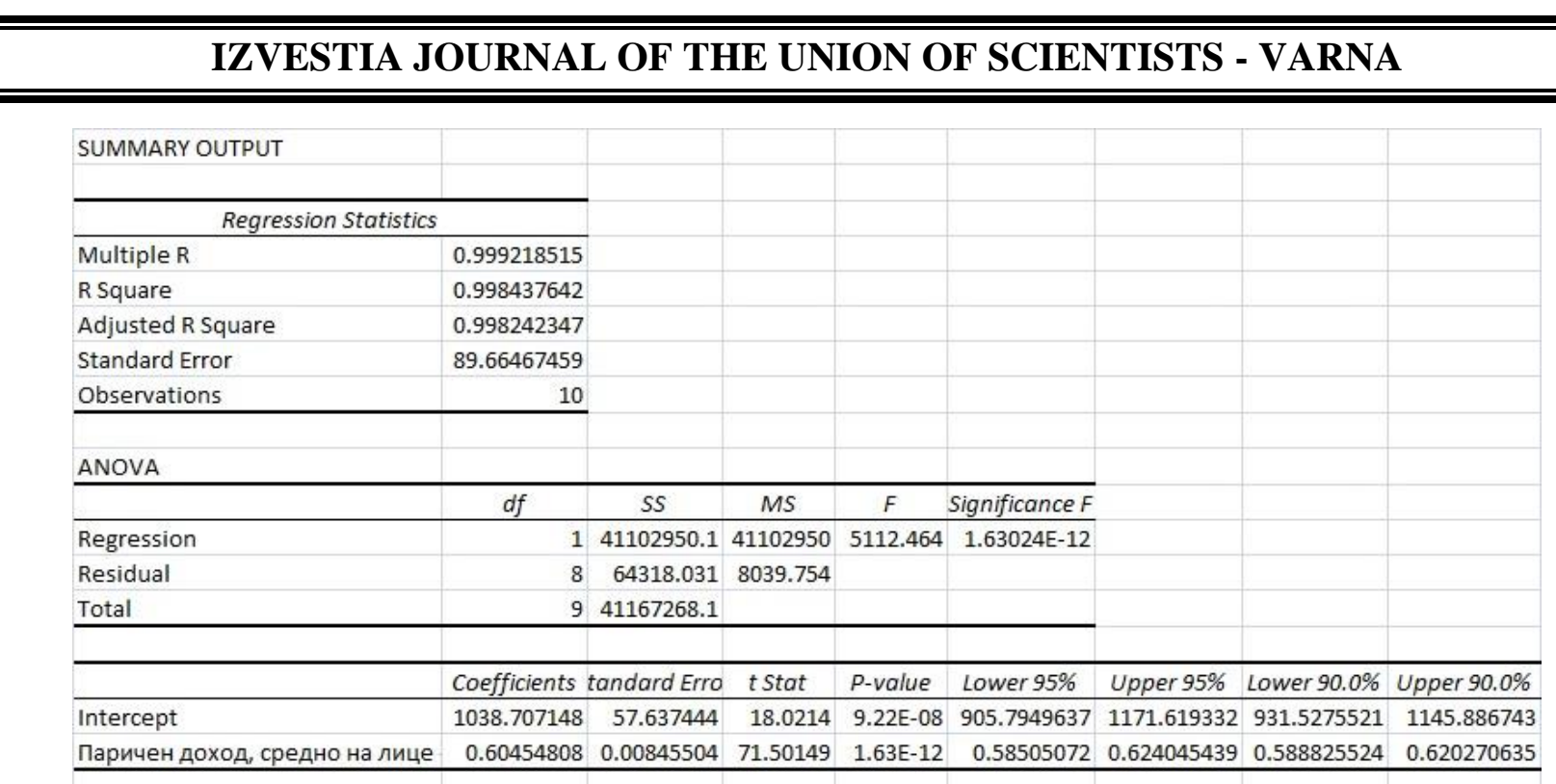

\section{Заключение}

Статията показва как с помощта на модула Data Analysis на MS Excel е възможно да се извърши един комплексен статистически анализ, демонстриран с примерите за доходите, разходите и потребителското търсене. Резултати от анализа съвпадат с изискванията на икономическата теория, а получените оценки са в границите на теоретичните очаквания.

Въпреки, че се налага да се пренареждат данни, да се разширяват изходните колони, да се преформатират графики и други, то анализите се реализират сравнително бързо и лесно и са достьпни за всички потребители на MS Excel. C всяка нова версия на MS Excel ce добавят по-нови и по-добри инструменти, като нови функции и диаграми за анализ на данни, но подобрения в Analysis ToolPak няма. Загадка е защо Microsoft не изразходва минималното време и средства, необходимо за обновяване и доразвитие на Analysis ToolPak.

\section{References}

1. HADZHIEV, V., DIMITROVA, V., LUBENOV, L. (2009) Statistical and Econometric Software, Varna: Science and Economics [in Bulgarian].

2. SALOV, V. et.al. (2019) Informatics, Varna: Science and Economics [in Bulgarian].

3. SLAVEVA, K., PETKOV, P., IVANOV, L., VARBANOV, T., and GEORGIEVA, N. (2016) Improving Statistics Training Using the Modern Information and Communication Technologies. Scientific Research Almanac, D. A. Tsenov Academy of Economics, Svishtov, Bulgaria, issue 23, pages 498-528 [in Bulgarian].

4. WINSTON, L. W. (2016) Microsoft Excel 2016. Data Analysis and Business Modeling. Washington: Microsoft Press. 